\title{
Dynamic Characteristics Analysis of Cylinders Bundle Coupling System Under Axial Flow
}

\author{
Ya-feng SHU***, Jian-jun WU*, Yong-wei YANG**, Wei-ming LIU**, Ke-wei TAO** \\ * Key Laboratory of Mechanics on Disaster and Environment in Western China, Ministry of Education, College of Civil \\ Engineering and Mechanics, Lanzhou University, Lanzhou 730000, China, E-mail: shuyafeng@impcas.ac.cn; \\ wujjun@lzu.edu.cn (Correspondingauthor) \\ **Institute of Modern Physics, Chinese Academy of Sciences, Lanzhou 730000, China, \\ E-mail: yangyongwei@impcas.ac.cn, liuweiming@impcas.ac.cn,taokewei@impcas.ac.cn \\ crossref http://dx.doi.org/10.5755/j02.mech.25101
}

\section{Introduction}

It is very important to study the dynamics characteristics of a CB system under axial flow for flow induced vibration(FIV) analysis of fuel rods and heat exchanger bundles in nuclear energy engineering [1] - [3]. Accurate calculation of FIV about CB can lay a foundation for predicting fretting wear and fatigue life [4], [5]. In a typical liquid metal fast neutron reactor (LMFR) fuel assembly design, the gap between fuel rods is very small, and the vibration of any rod will interact with the surrounding fuel rods due to fluid coupling. Chen S. S. established the additional mass coefficient model of the CB system in bounded domain [6], only considering the viscous damping coefficient of a single cylinder. Païdoussis established the model of fluid viscous coupling between cylinders [7], [8]. Liu studied the dynamic characteristics of the axial flow four-cylinder beam system through the coupling of computational fluid dynamics (CFD) and computational solid dynamics (CSD), and the calculated critical velocity was similar to that of the simplified Païdoussis model [9]. But their work is mainly to establish the dynamic model of the cylinders bundle under steady flow and to carry out the modal analysis and system stability analysis. In practical engineering, the flow field is not steady flow, due to the influence of the primary pump speed, it will produce the inlet pulsating flow which will lead to the fretting wear and vibration fatigue. Jin J. D., Wang L., et al., studied the dynamics of the pipes conveying pulsating flow [10], [11], Païdoussis is the only one who studied the effect of pulsating parameters on the single cylinder [12], but few scholars have been involved in the study of the CB subjected to axial pulsating outflow. Therefore, it is necessary to study the dynamic characteristics of pulsating flow of the CB system.

This paper is based on Chen and Païdoussis idea to establish the dynamic model of the CB system. Considering the pulsating flow caused by the pump speed, the mathematical model of the CB vibration is established. Then the correctness of the model and the numerical computational code is verified. Taking the coolant parameters of MYRRHA lead-bismuth reactor and the geometric parameters of CB as examples, the effects of equivalent mass and slenderness ratio on the critical flow velocity are analyzed. Finally, the dynamic characteristics of the 7-CB system are calculated. The effects of the pulsating flow parameters on the amplitude of the forced vibration CB system are analyzed through the displacement time history, the trajectory of the midspan cross section and the amplitude-frequency characteristics.

\section{Establishment of the dynamic equation of a CB cou- pling system}

The fuel rod is mainly composed of slender cladding tube and pellets in the reactor, the fuel assembly can be simplified as a CB system consisting of $K$ vertical parallel slender elastic cylinders with identical radius $R$ in bounded domain, as shown in Fig. 1. So each fuel rod is treated as a Euler-Bernoulli beam. The coolant fluid flows from the bottom to the top passing through the $\mathrm{CB}$, and the pulsating velocity caused by the pump is expressed as $U=U_{0}(1+\mu \sin \Omega t)$, assuming the pulsating parameters $\mu$ is sufficiently small for the flow velocity to remain essentially uniform in the flow channel [12]. To aid discussion, the parameters $G_{c}$ and $G_{w}$ are defined such that $G_{c}$ means the ratio of the smallest inter-cylinder gap to the cylinder radius $R$, and $G_{w}$ means the ratio of the smallest cylinder-to-channel wall gap to the cylinder radius $R$. Noting that the coordinate axial direction is different from Païdoussis M. P., et al. [13] because the coolant flow direction in general reactor is upward.
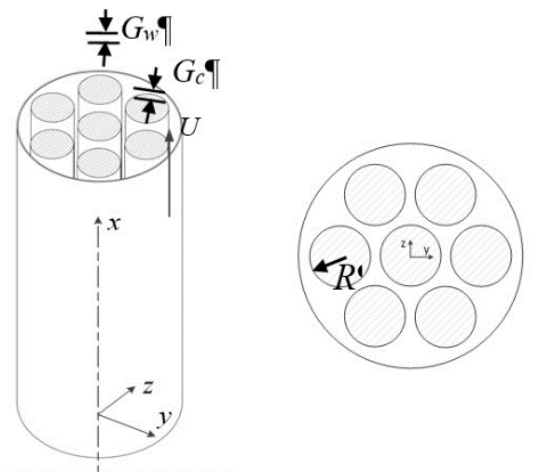

Fig. 1 The schematic diagram of a CB system in flow

Let us consider the small lateral motion of any one cylinder of the CB system, and assume that the angle of deformation incidence is small enough so that no flow separation occurs. The fluid force acting on the structure is obtained by solving Navier Stokes equation, but the solution process is complex and difficult. For calculation convenience, Païdoussis M. P. and Chen S. S. clearly divided the fluid force into three part: inviscid force, frictional force and hydrostatic force [14], [15]. At first, we discuss the balance relation in the xoy plane. The force and moment acting on an element $\delta x$ are shown in Fig. 2, the force balance equations of cylinder $j$ in the $x$-direction and y-direction can be written as: 


$$
\begin{gathered}
\frac{\partial T}{\partial x}-m g+F-(F+F) \frac{\partial y}{\partial x}+F=0 \\
\frac{\partial Q_{j}}{\partial x}-\left(F_{A j}^{y}+F_{N j}^{y}\right)+F_{P j}^{y}+F_{L j} \frac{\partial y_{j}}{\partial x}+\frac{\partial}{\partial x}\left(T_{j} \frac{\partial y_{j}}{\partial x}\right)- \\
-m \frac{\partial^{2} y_{j}}{\partial t^{2}}=0
\end{gathered}
$$

and the relation between the shear force and the moment is written as:

$$
Q_{j}=-\frac{\partial M^{*}}{\partial x}=-\left(E+E^{*} \frac{\partial}{\partial t}\right) I \frac{\partial^{3} y}{\partial x^{3}},
$$

where the fact that $y$ and its derivatives are small has been utilized. And it has been assumed that the material of cylinder is viscoelastic and consistent with Kelvin-Voigt hypothesis, namely, $\sigma=\left(E+E^{*} \partial / \partial t\right) \varepsilon$. The material viscoelastic coefficient is $E^{*}$, the modulus of elasticity is $E$, the cross-section moment of inertia is $I$.

Based on Lighthill slender body theory and unsteady Bernoulli equation [15, 16], the inviscid hydrodynamic force is expressed as:

$$
F_{A j}^{y}=-\oint_{S} \rho\left(\frac{\partial}{\partial t}+U \frac{\partial}{\partial x}\right) \phi j d s
$$

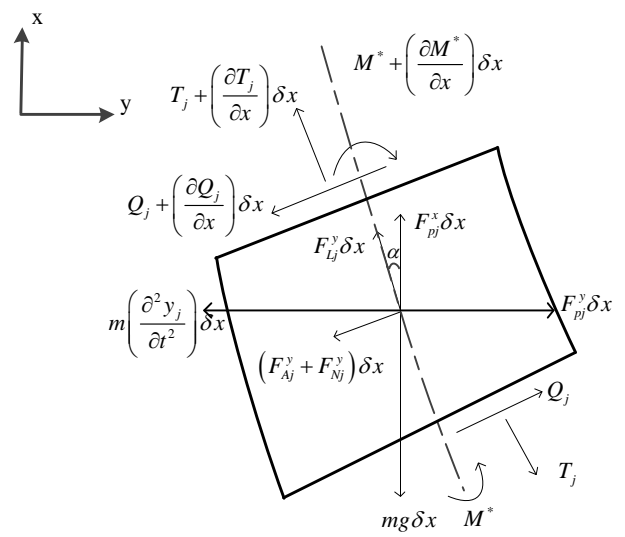

Fig. 2 Cylindrical element force analysis.

where: $\rho$ is the fluid density, $\phi$ is the velocity potential of the CB system. $j$ is the unit vector in the direction of the displacement $v$, and in Eq. (4), $U$ is the axial flow velocity which does not necessarily require constant velocity according to the Lighthill theory and unsteady Bernoulli theory. S. Suss [19] derived the added mass coefficients and viscous hydrodynamic coefficients computational method and obtained the relation between the inviscid hydrodynamic force and the displacement [18]. It can be expressed as follows:

$$
\begin{aligned}
& F_{A j}^{y}=-\rho \pi R^{2} \sum_{l=1}^{K}\left[\varepsilon_{j l} \frac{D^{2} y_{l}}{D t^{2}}+e_{j l} \frac{D^{2} z_{l}}{D t^{2}}\right], \\
& F_{A j}^{z}=-\rho \pi R^{2} \sum_{l=1}^{K}\left[\kappa_{j l} \frac{D^{2} y_{l}}{D t^{2}}+k_{j l} \frac{D^{2} z_{l}}{D t^{2}}\right] .
\end{aligned}
$$

and the viscous hydrodynamic force is written as:

$$
\begin{aligned}
& F_{N j}^{y}=\frac{1}{2} \rho D U C_{f} \sum_{l=1}^{K}\left[\zeta_{j l} \frac{D y_{l}}{D t}+g_{j l} \frac{D z_{l}}{D t}\right]+ \\
& +\frac{1}{2} \rho D U C_{D} \sum_{l=1}^{K}\left[\zeta_{j l} \frac{\partial y_{l}}{\partial t}+g_{j l} \frac{\partial z_{l}}{\partial t}\right], \\
& F_{N j}^{z}=\frac{1}{2} \rho D U C_{f} \sum_{l=1}^{K}\left[\sigma_{j l} \frac{D y_{l}}{D t}+s_{j l} \frac{D z_{l}}{D t}\right]+ \\
& +\frac{1}{2} \rho D U C_{D} \sum_{l=1}^{K}\left[\sigma_{j l} \frac{\partial y_{l}}{\partial t}+s_{j l} \frac{\partial z_{l}}{\partial t}\right],
\end{aligned}
$$

where: $D$ is the diameter of cylinder, $C_{f}$ is the frictional coefficient, $C_{D}$ is the pressure drag coefficient, and the material derivative $D / D t=\partial / \partial t+U(\partial / \partial x)$. The added mass coefficients $\varepsilon_{j l}, e_{j l}, \kappa_{j l}, k_{j l}$ and the viscous coupling coefficients $\zeta_{j l}, g_{j l}, \sigma_{j l}, s_{j l}$ is derived by Suss S. in detail [18].

After the right sides of Eq. (5) and Eq. (6) expanded, the pulsating flow will produce an added inviscid hydrodynamic term in the $y$ direction and $z$ direction as:

$$
\begin{aligned}
& { }^{a d d} F_{A j}^{y}=-\rho \pi R^{2} \sum_{l=1}^{K}\left[\varepsilon_{j l} \dot{U} \frac{\partial y_{l}}{\partial x}+e_{j l} \dot{U} \frac{\partial z_{l}}{\partial x}\right], \\
& { }^{a d d} F_{A j}^{z}=-\rho \pi R^{2} \sum_{l=1}^{K}\left[\kappa_{j l} \dot{U} \frac{\partial y_{l}}{\partial x}+k_{j l} \dot{U} \frac{\partial z_{l}}{\partial x}\right],
\end{aligned}
$$

noting that the added term has been included in the Eq. (5) and Eq. (6), respectively. Here it is to illustrate the effect of pulsating flow.

The steady pressure force can be obtained through considering the sum of all forces acting on an imagined isolated element, "frozen" in instant time, summing the forces shown in Fig. 3, which is equal to the buoyancy force, we obtain:

$$
\begin{aligned}
& {\left[-\frac{\partial(P A)}{\partial x}+F_{p j}^{x}\right] \delta x i+\left[F_{p j}^{y}-\frac{\partial}{\partial x}\left(P A \frac{\partial y_{j}}{\partial x}\right)\right] \delta x j=} \\
& =-\int \grave{\rho} P \hat{\mathbf{n}} d A=-\iiint_{v o l} \nabla P d(\mathrm{vol})=-\frac{\partial P}{\partial x} A \delta x i,
\end{aligned}
$$

where: $P$ is the steady pressure neglecting the fluctuating pressure, $\overrightarrow{\boldsymbol{\imath}}, \overrightarrow{\boldsymbol{J}}$ is the unit vector in the $x$ and $y$ direction, respectively.

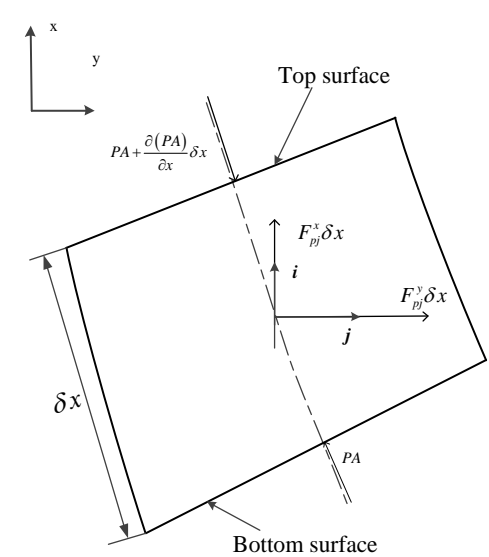

Fig. 3 The external resultant force of equivalent "rigid body" element 
Through Eq. (11), the hydrostatic pressure force can be written as:

$$
\begin{aligned}
& F_{p j}^{x}=-\frac{\partial P}{\partial x} A+\frac{\partial(P A)}{\partial x}=P \frac{\partial A}{\partial x}=0 \\
& F_{p j}^{y}=\frac{\partial}{\partial x}\left(P A \frac{\partial y_{j}}{\partial x}\right)=A \frac{\partial P}{\partial x} \frac{\partial y_{j}}{\partial x}+P A \frac{\partial^{2} y_{j}}{\partial x^{2}}
\end{aligned},
$$

where: $A$ is the cross-sectional area, a constant, $\partial A / \partial x=0$. Substituting Eq. (12) into Eq. (1) and Eq. (2), respectively, then Eq. (1) and Eq. (2) are written as:

$$
\begin{aligned}
& \frac{\partial T_{j}}{\partial x}-m g+F_{L j}-\left(F_{A j}^{y}+F_{N j}^{y}\right) \frac{\partial y_{j}}{\partial x}=0 \\
& \frac{\partial Q_{j}}{\partial x}-\left(F_{A j}^{y}+F_{N j}^{y}\right)+A \frac{\partial P}{\partial x} \frac{\partial y_{j}}{\partial x}+P A \frac{\partial^{2} y_{j}}{\partial x^{2}}+ \\
& +F_{L j} \frac{\partial y_{j}}{\partial x}+\frac{\partial}{\partial x}\left(T \frac{\partial y_{j}}{\partial x}\right)-m \frac{\partial^{2} y_{j}}{\partial t^{2}}=0 .
\end{aligned}
$$

The viscous fluid force is given by G. Taylor [18] through experiment, as follows:

$$
\begin{aligned}
& F_{N j}^{y}=\frac{1}{2} \rho D U^{2}\left(C_{f} \sin \alpha+C_{D p} \sin ^{2} \alpha\right), \\
& F_{L j}^{y}=\frac{1}{2} \rho D U^{2} C_{f} \cos \alpha .
\end{aligned}
$$

According to small deformation hypothesis, Eq. (15) and Eq. (16) can be linearized [13], then both can be reduced to:

$$
\begin{aligned}
& F_{N j}^{y}=\frac{1}{2} \rho D U C_{f}\left(\frac{\partial y_{j}}{\partial t}+U \frac{\partial y_{j}}{\partial x}\right)+\frac{1}{2} \rho D C_{D} \frac{\partial y_{j}}{\partial t}, \\
& F_{L j}=\frac{1}{2} \rho D U^{2} C_{f},
\end{aligned}
$$

where: $C_{f}$ is the frictional drag coefficients, respectively. $C_{D}$ is the shape drag coefficient at zero flow velocity.

According to the small deformation theory, assuming the lateral displacement is one order small quantity, i.e., $y$ : $o(\varepsilon)$. Then, the rotation angle, bending moment and shear force are all first order small quantities. So $F_{A j}^{y}+F_{N j}^{y}: o\left(\varepsilon^{2}\right)$. Neglecting the second order small quantity, Eq.(13) can be written as:

$$
\frac{\partial T_{j}}{\partial x}-m g+F_{L j}=0
$$

The Eq. (15) can be integrated from $\mathrm{x}$ to $L$ (where $L$ is the length of cylinder), we can obtain:

$$
T_{j}(x)=T(L)-m g(L-x)+\int_{x}^{L} F_{L j} d x,
$$

where: $T(L)$ is the tension acting on the downstream end of the cylinder. In 2005, Modarres-Sadeghi gave the latest expression [19] as follows:

$$
\begin{aligned}
& T(L)=\bar{T} \delta+\frac{1}{2} \rho D^{2} U^{2} C_{b}(1-\delta)- \\
& -\frac{L}{2} \delta\left(\frac{1}{2} \rho D U^{2} C_{f}\left(1+\frac{D}{D_{h}}\right)-m g\right),
\end{aligned}
$$

where: $\bar{T}$ is externally applied uniform tension, $C_{\mathrm{b}}$ is the base drag force coefficient.

Finally, to complete the Eq. (3) we need a workable expression of $d p / d x$. This is also required in the formulation of the pressure force Eq. (12). A simple expression of $d p / d x$ has been derived by Païdoussis M. P. (1973) by examining the axial force balance over the total channel surface area of a small element. According to the axial force balance, i.e., the 1-D unsteady modified Euler equation, assuming the lateral movement of the cylinders to have negligible effect on the axial pressure distribution. So we obtain:

$$
-A_{c h} \frac{\partial P}{\partial x}-F_{f}-\rho g A_{c h}-\rho A_{c h} \frac{d U}{d t}=0,
$$

where: $A_{c h}$ is the total cross-sectional flow area of the fluid volume element, $F_{f}$ is the frictional force per unit length, $g$ is the gravity acceleration.

Now, assuming a constant frictional force per unit area of the surface, the side area of the cylinder per unit length is $S=\pi D$. We can write:

$$
F_{f}=F_{L j}\left(\frac{S_{t o t}}{S}\right)
$$

where: $F_{L j}$ is the longitude viscous hydrodynamic force. Defining a hydraulic diameter, $D_{h}=4 A_{\mathrm{ch}} / S_{\text {tot }}$. And utilizing the expression of $F_{L j}$ in Eq. (18), we obtain:

$$
A \frac{\partial P}{\partial x}=-\rho g A-\frac{1}{2} \rho D U^{2} C_{f}\left(\frac{D}{D_{h}}\right)-\rho A \frac{d U}{d t},
$$

integrating Eq. (24) from $x$ to $L$, we obtain:

$$
\begin{aligned}
& A P(x)=A P(L)+\left[\frac{1}{2} \rho D U^{2} C_{f}\left(\frac{D}{D_{h}}\right)+\rho g A+\right. \\
& \left.+\rho A \frac{d U}{d t}\right](L-x) .
\end{aligned}
$$

Modarres-Sadeghi gives the expression of the pressure force at the downstream end of the cylinder as follows [19]:

$$
A p(L)=\left[(1-2 v) \bar{p} A-\rho g A \frac{L}{2}\right] \delta
$$

where: $\bar{p}$ represents the pressure at $x=1 / 2 L$ cross-section. $\delta=1$ means the downstream end is fixed and 0 means the downstream end is free to slide. 
Let the transverse deformation of the jth cylinder be $v j+w k$, Substituting Eqs. (3), (17), (18), (24) and (25) into Eq. (14) and applying the external excitation $f_{j e}$ on the $j$ th cylinder, we can obtain the motion equation of the $j$ th cylinder as follows:

$$
\begin{aligned}
& E^{*} I \frac{\partial^{5} v_{j}}{\partial t \partial x^{4}}+E I \frac{\partial^{4} v_{j}}{\partial x^{4}}+F_{A j}^{y}+F_{N j}^{y}- \\
& -\{\delta[\overline{\mathrm{T}}+(1-2 v) \bar{P} A]+ \\
& +\left[\frac{1}{2} \rho U^{2} C_{f}\left(1+\frac{D}{D_{h}}\right)-(m-\rho A) g+\rho A \frac{d U}{d t}\right] \\
& \left.\left[\left(1-\frac{1}{2} \delta\right) L-x\right]+\frac{1}{2} \rho D^{2} U^{2} C_{b}(1-\delta)\right\} \frac{\partial^{2} v_{j}}{\partial x^{2}}+ \\
& +\left[-(m-\rho A) g+\frac{1}{2} \rho D U^{2} C_{f}\left(\frac{D}{D_{h}}\right)+\rho A \frac{d U}{d t}\right] \frac{\partial v_{j}}{\partial x} \\
& +m \frac{\partial^{2} v_{j}}{\partial t^{2}}=f_{j}^{e},
\end{aligned}
$$

Similarly, the $z$ direction has the same equation of motion with respect to $w_{j}$. The dimensionless parameters are defined as below:

$$
\begin{aligned}
& \xi=\frac{x}{L}, \tau=\left(\frac{E I}{m+\rho A}\right)^{1 / 2} \frac{t}{L^{2}}, \omega=\left(\frac{m+\rho A}{E I}\right)^{1 / 2} \Omega L^{2}, \\
& \varepsilon=\frac{L}{D}, \beta=\frac{\rho A}{m+\rho A}, \gamma=\frac{(m-\rho A) g L^{3}}{E I}, h=\frac{D}{D_{h}}, \\
& c=\frac{4}{\pi} C_{D}\left(\frac{\rho A}{E I}\right)^{1 / 2} L, c_{b}=\frac{4}{\pi} C_{b}, \Pi=\frac{\bar{P} A L^{2}}{E I}, \Gamma=\frac{\bar{T} L^{2}}{E I}, \\
& \alpha=\left(\frac{I}{E(m+\rho A)}\right)^{1 / 2} \frac{E^{*}}{L^{2}}, c_{f}=\frac{4}{\pi} C_{f}, u=\sqrt{\frac{\rho A}{E I}} U L, \\
& f_{j}=\frac{L^{3}}{E I} f_{j}^{e}, u_{0}=\sqrt{\frac{\rho A}{E I}} U_{0} L, \eta_{j}=\frac{v_{j}}{L}, \\
& \text { when } j=1,2, \ldots, K, \text { and } \eta_{j}=\frac{w_{j-K}}{L}, \\
& \text { where } j=K+1, K+2, \ldots, 2 K .
\end{aligned}
$$

For the convenience of calculation, the equation is dimensionless by using Eq. (28), the dynamic equation Eq. (27) of a CB system is written in the form of matrix as follows:

$$
\begin{aligned}
& \boldsymbol{A} \frac{\partial^{5} \boldsymbol{\eta}}{\partial \xi^{4} \partial \tau}+\boldsymbol{I} \frac{\partial^{4} \boldsymbol{\eta}}{\partial \xi^{4}}+\left(\boldsymbol{C}+\boldsymbol{C}_{1}\right) \frac{\partial^{2} \boldsymbol{\eta}}{\partial \xi \partial \tau}+\left(\boldsymbol{E}+\boldsymbol{E}_{1}\right) \frac{\partial^{2} \boldsymbol{\eta}}{\partial \xi^{2}}+\left(\boldsymbol{F}+\boldsymbol{F}_{1}\right) \xi \frac{\partial^{2} \boldsymbol{\eta}}{\partial \xi^{2}}+\left(\boldsymbol{G}+\boldsymbol{G}_{1}\right) \frac{\partial \boldsymbol{\eta}}{\partial \xi}+ \\
& +\left(\boldsymbol{H}+\boldsymbol{H}_{1}\right) \frac{\partial \boldsymbol{\eta}}{\partial \tau}+\boldsymbol{M} \frac{\partial^{2} \boldsymbol{\eta}}{\partial \tau^{2}}=\boldsymbol{f} .
\end{aligned}
$$

Here is the matrix expression in Eq. (29):

$\boldsymbol{A}=\alpha \boldsymbol{I}$, where $\boldsymbol{I}$ is the unit matrix.

$$
\begin{aligned}
& \boldsymbol{C}=-2 \beta^{1 / 2} u_{0} \boldsymbol{M}_{a}, \boldsymbol{C}_{1}=-2 \beta^{1 / 2} u_{0} \mu \sin \omega \tau \boldsymbol{M}_{a}, \\
& \boldsymbol{E}=-u_{0}^{2} \boldsymbol{M}_{a}-\left\{\delta[\Gamma+(1-2 v) \Pi]+(1-\delta) \frac{1}{2} c_{b} u_{0}^{2}+\left(1-\frac{1}{2} \delta\right)\left[\frac{1}{2} \varepsilon c_{f} u_{0}^{2}(1+h)-\gamma\right]\right\} \boldsymbol{I}, \\
& \boldsymbol{E}_{1}=-u_{0}^{2}\left(2 \mu \sin \omega \tau+\mu^{2} \sin ^{2} \omega \tau\right) \boldsymbol{M}_{a}-u_{0}^{2}\left(2 \mu \sin \omega \tau+\mu^{2} \sin ^{2} \omega \tau\right)\left\{\frac{1}{2}(1-\delta) c_{b}+\right. \\
& \left.+\left(1-\frac{1}{2} \delta\right)\left[\frac{1}{2} \varepsilon c_{f}(1+h)\right]\right\} \boldsymbol{I}+\beta^{1 / 2} \mu u_{0}\left(1-\frac{1}{2} \delta\right) \cos \omega \tau \boldsymbol{I}, \\
& \boldsymbol{F}=\left[\frac{1}{2} \varepsilon c_{f} u_{0}^{2}(1+h)-\gamma\right] \boldsymbol{I}, \boldsymbol{F}_{1}=u_{0}^{2}\left(2 \mu \sin \omega \tau+\mu^{2} \sin ^{2} \omega \tau\right)\left[\frac{1}{2} \varepsilon c_{f}(1+h)\right] \boldsymbol{I}-\beta^{1 / 2} \mu u_{0} \cos \omega \tau \boldsymbol{I}, \\
& \boldsymbol{G}=\frac{1}{2} \varepsilon c_{f} u^{2}(1+h) \boldsymbol{C}_{v}+\left[\frac{1}{2} \varepsilon c_{f} u^{2}(1+h)-\gamma\right] \boldsymbol{I}, \\
& \boldsymbol{G}_{1}=u_{0}^{2}\left(2 \mu \sin \omega \tau+\mu^{2} \sin ^{2} \omega \tau\right)\left[\frac{1}{2} \varepsilon c_{f}(1+h)\right]\left(\boldsymbol{C}_{v}+\boldsymbol{I}\right)+\mu u_{0} \beta^{1 / 2} \cos \omega \tau\left(\boldsymbol{M}_{a}+\boldsymbol{I}\right), \\
& \boldsymbol{H}=\left(\frac{1}{2} \varepsilon c_{f} \beta^{1 / 2} u+\frac{1}{2} \varepsilon c \beta^{1 / 2}\right) \boldsymbol{C}_{v}, \boldsymbol{H}_{1}=u_{0} \mu \sin \omega \tau\left(\frac{1}{2} \varepsilon c_{f} \beta^{1 / 2}\right) \boldsymbol{C}_{v}, \boldsymbol{M}=-\beta \boldsymbol{M}_{a}+(1-\beta) \boldsymbol{I},
\end{aligned}
$$

where the added mass coefficient matrix and viscous coupling coefficient matrix are as follows:

$$
\boldsymbol{M}_{a}=\left[\begin{array}{ll}
{\left[\varepsilon_{j l}\right]} & {\left[e_{j l}\right]} \\
{\left[\kappa_{j l}\right]} & {\left[k_{j l}\right]}
\end{array}\right], \boldsymbol{C}_{v}=\left[\begin{array}{ll}
{\left[\zeta_{j l}\right]} & {\left[g_{j l}\right]} \\
{\left[\sigma_{j l}\right]} & {\left[s_{j l}\right]}
\end{array}\right],
$$

where: the reference [17] is for the detailed calculation of $\boldsymbol{M}_{\mathrm{a}}$ and $\boldsymbol{C}_{\mathrm{v}}$. The external excitation is $\boldsymbol{f}=\left[f_{1}, f_{2}, \cdots, f_{2 K}\right]^{T}$.

\section{Mathematic method}

Partial differential Eq. (29) is transformed into or- 
dinary differential equation by separating variables. Generally, Galerkin method is adopted to discrete Eq. (29), and the displacement can be written as follows

$$
\begin{aligned}
& \eta_{j}(\xi, \tau)=\sum_{i=1}^{\infty} \phi_{i}(\xi) q_{i}^{j}(\tau), \\
& \boldsymbol{A} \sum_{i=1}^{\infty} \dot{\boldsymbol{q}}_{i} \int_{0}^{1} \frac{d^{4} \phi_{i}}{d \xi^{4}} \phi_{r} d \xi+\boldsymbol{I} \sum_{i=1}^{\infty} \boldsymbol{q}_{i} \int_{0}^{1} \frac{d^{4} \phi_{i}}{d \xi^{4}} \phi_{r} d \xi+\left(\boldsymbol{C}+\boldsymbol{C}_{1}\right) \sum_{i=1}^{\infty} \dot{\boldsymbol{q}}_{i} \int_{0}^{1} \frac{d \phi_{i}}{d \xi} \phi_{r} d \xi+\left(\boldsymbol{E}+\boldsymbol{E}_{1}\right) \sum_{i=1}^{\infty} \boldsymbol{q}_{i} \int_{0}^{1} \frac{d^{2} \phi_{i}}{d \xi^{2}} \phi_{r} d \xi+ \\
& +\left(\boldsymbol{F}+\boldsymbol{F}_{1}\right) \sum_{i=1}^{\infty} \boldsymbol{q}_{i} \int_{0}^{1} \xi \frac{d^{2} \phi_{i}}{d \xi^{2}} \phi_{r} d \xi+\left(\boldsymbol{G}+\boldsymbol{G}_{1}\right) \sum_{i=1}^{\infty} \boldsymbol{q}_{i} \int_{0}^{1} \frac{d \phi_{i}}{d \xi} \phi_{r} d \xi+\left(\boldsymbol{H}+\boldsymbol{H}_{1}\right) \sum_{i=1}^{\infty} \dot{\boldsymbol{q}}_{i} \int_{0}^{1} \phi_{i} \phi_{r} d \xi+\boldsymbol{M} \sum_{i=1}^{\infty} \ddot{\boldsymbol{q}}_{i} \int_{0}^{1} \phi_{i} \phi_{r} d \xi=\int_{0}^{1} \boldsymbol{f} \phi_{r} d \xi,
\end{aligned}
$$

where: the generalized coordinates are $\boldsymbol{q}_{i}=\left[q_{i}^{1}, q_{i}^{2}, \cdots q_{i}^{2 K}\right]^{T}, i, r=1,2,3, \ldots, \infty$. where: $\phi_{i}$ is the shape function of the beam satisfying boundary conditions, and $q_{i}^{j}$ is the corresponding generalized coordinate. So let's substitute Eq. (32) into Eq. (29), multiplying by $\phi_{r}$ and then integrating $\xi=0$ to 1 , so we get the Eq.

$$
\begin{aligned}
& \boldsymbol{A} \lambda_{r}^{4} \delta_{r i} \dot{\boldsymbol{q}}_{r}+\boldsymbol{I} \lambda_{r}^{4} \delta_{r i} \boldsymbol{q}_{r}+\boldsymbol{C} \sum_{i=1}^{\infty} a_{r i} \dot{\boldsymbol{q}}_{i}+\boldsymbol{C}_{1} \sum_{i=1}^{\infty} a_{r i} \dot{q}_{i}+\boldsymbol{E} \sum_{i=1}^{\infty} b_{r i} \boldsymbol{q}_{i}+\boldsymbol{E}_{1} \sum_{i=1}^{\infty} b_{r i} \boldsymbol{q}_{i}+\boldsymbol{F} \sum_{i=1}^{\infty} d_{r i} \boldsymbol{q}_{i}+ \\
& +\boldsymbol{F}_{1} \sum_{i=1}^{\infty} d_{r i} q_{i}+\boldsymbol{G} \sum_{i=1}^{\infty} a_{r i} q_{i}+\boldsymbol{G}_{1} \sum_{i=1}^{\infty} a_{r i} q_{i}+\boldsymbol{H} \delta_{r i} \dot{q}_{r}+\boldsymbol{H}_{1} \delta_{r i} \dot{q}_{r}+\boldsymbol{M} \delta_{r i} \ddot{q}_{r}=\tilde{\boldsymbol{Q}},
\end{aligned}
$$

where: $\lambda_{r}$ is the $r$ th order eigenvalue corresponding to the beam equation. $\delta_{i j}$ is the Kronecker symbol. In Eq. (34), the expressions of $a_{r i}, b_{r i}$ and $c_{r i}$ are written as:

$$
a_{r i}=\int_{0}^{1} \frac{d \phi_{i}}{d \xi} \phi_{r} d \xi, b_{r i}=\int_{0}^{1} \frac{d^{2} \phi_{i}}{d \xi^{2}} \phi_{r} d \xi, d_{r i}=\int_{0}^{1} \xi \frac{d^{2} \phi_{i}}{d \xi^{2}} \phi_{r} d \xi .
$$

The specific integral values of $a_{r i}, b_{r i}$ and $d_{r i}$ under three classical boundary conditions are in reference [21]. In general, the Eq. (34) can be written in matrix form as follows:

$$
\tilde{\boldsymbol{M}} \ddot{\boldsymbol{q}}+\tilde{\boldsymbol{C}} \dot{\boldsymbol{q}}+\tilde{\boldsymbol{C}}_{1}(\tau) \boldsymbol{q}+\tilde{\boldsymbol{K}} \boldsymbol{q}+\tilde{\boldsymbol{K}}_{1}(\tau) \boldsymbol{q}=\tilde{\boldsymbol{Q}}
$$

where the discrete truncation number of Galerkin is $N=3$ in the paper and $\boldsymbol{q}=\left[\boldsymbol{q}_{1}, \boldsymbol{q}_{2}, \cdots, \boldsymbol{q}_{N}\right]$. The coefficient matrix dimension of Eq. (36) is $2 K N \times 2 K N$, the coefficient matrix expressions are as follows:

$$
\begin{aligned}
& \tilde{\boldsymbol{M}}=\left[\begin{array}{cccc}
\boldsymbol{M} & \mathbf{0} & \cdots & \mathbf{0} \\
\mathbf{0} & \boldsymbol{M} & & \\
\vdots & & \ddots & \\
\mathbf{0} & \mathbf{0} & \cdots & \boldsymbol{M}
\end{array}\right] \tilde{\boldsymbol{C}}=\left[\begin{array}{cccc}
\boldsymbol{A} \lambda_{1}^{4} & \mathbf{0} & \cdots & \mathbf{0} \\
\mathbf{0} & \boldsymbol{A} \lambda_{2}^{4} & & \\
\vdots & & \ddots & \\
\mathbf{0} & \mathbf{0} & \cdots & \boldsymbol{A} \lambda_{N}^{4}
\end{array}\right]+\left[\begin{array}{cccc}
\boldsymbol{C} a_{11} & \boldsymbol{C} a_{12} & \cdots & \boldsymbol{C} a_{1 N} \\
\boldsymbol{C} a_{21} & \boldsymbol{C} a_{22} & & \\
\vdots & & \ddots & \\
\boldsymbol{C} a_{N 1} & \boldsymbol{C} a_{N 2} & \cdots & \boldsymbol{C} a_{N N}
\end{array}\right]+\left[\begin{array}{cccc}
\boldsymbol{H} & \mathbf{0} & \cdots & \mathbf{0} \\
\mathbf{0} & \boldsymbol{H} & & \\
\vdots & & \ddots & \\
\mathbf{0} & \mathbf{0} & \cdots & \boldsymbol{H}
\end{array}\right], \\
& \tilde{\boldsymbol{C}}_{1}(\tau)=\beta^{1 / 2} u_{0} \mu \sin \omega \tau\left(\frac{1}{2} \varepsilon c_{f}\left[\begin{array}{cccc}
\boldsymbol{C}_{v} & \mathbf{0} & \cdots & \mathbf{0} \\
\mathbf{0} & \boldsymbol{C}_{v} & & \\
\vdots & & \ddots & \\
\mathbf{0} & \mathbf{0} & \cdots & \boldsymbol{C}_{v}
\end{array}\right]-2\left[\begin{array}{cccc}
\boldsymbol{M}_{a} a_{11} & \boldsymbol{M}_{a} a_{12} & \cdots & \boldsymbol{M}_{a} a_{1 N} \\
\boldsymbol{M}_{a} a_{21} & \boldsymbol{M}_{a} a_{22} & & \\
\vdots & & \ddots & \\
\boldsymbol{M}_{a} a_{N 1} & \boldsymbol{M}_{a} a_{N 2} & \cdots & \boldsymbol{M}_{a} a_{N N}
\end{array}\right]\right) \text {, } \\
& \tilde{\boldsymbol{K}}=\left[\begin{array}{cccc}
\boldsymbol{I} \lambda_{1}^{4} & \mathbf{0} & \cdots & \mathbf{0} \\
\mathbf{0} & \boldsymbol{I} \lambda_{2}^{4} & & \\
\vdots & & \ddots & \\
\mathbf{0} & \mathbf{0} & \cdots & \boldsymbol{I} \lambda_{N}^{4}
\end{array}\right]+\left[\begin{array}{cccc}
\boldsymbol{E} b_{11} & \boldsymbol{E} b_{12} & \cdots & \boldsymbol{E} b_{1 N} \\
\boldsymbol{E} b_{21} & \boldsymbol{E} a_{22} & & \\
\vdots & & \ddots & \\
\boldsymbol{E} b_{N 1} & \boldsymbol{E} b_{N 2} & \cdots & \boldsymbol{E} b_{N N}
\end{array}\right]+\left[\begin{array}{cccc}
\boldsymbol{F} d_{11} & \boldsymbol{F} d_{12} & \cdots & \boldsymbol{F} d_{1 N} \\
\boldsymbol{F} d_{21} & \boldsymbol{F} d_{22} & & \\
\vdots & & \ddots & \\
\boldsymbol{F} d_{N 1} & \boldsymbol{F} d_{N 2} & \cdots & \boldsymbol{F} d_{N N}
\end{array}\right]+\left[\begin{array}{cccc}
\boldsymbol{G} a_{11} & \boldsymbol{G} a_{12} & \cdots & \boldsymbol{G} a_{1 N} \\
\boldsymbol{G} a_{21} & \boldsymbol{G} a_{22} & & \\
\vdots & & \ddots & \\
\boldsymbol{G} a_{N 1} & \boldsymbol{G} a_{N 2} & \cdots & \boldsymbol{G} a_{N N}
\end{array}\right], \\
& \tilde{\boldsymbol{K}}_{1}(\tau)=u_{0}^{2} \mu \sin \omega \tau(2+\mu \sin \omega \tau)\left(-\left[\begin{array}{cccc}
\left(\boldsymbol{M}_{a}+p \boldsymbol{I}\right) b_{11} & \left(\boldsymbol{M}_{a}+p \boldsymbol{I}\right) b_{12} & \cdots & \left(\boldsymbol{M}_{a}+p \boldsymbol{I}\right) b_{1 N} \\
\left(\boldsymbol{M}_{a}+p \boldsymbol{I}\right) b_{21} & \left(\boldsymbol{M}_{a}+p \boldsymbol{I}\right) b_{22} & & \\
\vdots & & \ddots & \\
\left(\boldsymbol{M}_{a}+p \boldsymbol{I}\right) b_{N 1} & \left(\boldsymbol{M}_{a}+p \boldsymbol{I}\right) b_{N 2} & \cdots & \left(\boldsymbol{M}_{a}+p \boldsymbol{I}\right) b_{N N}
\end{array}\right]+\right. \\
& \left.+\frac{1}{2} \varepsilon c_{f}(1+h)\left[\begin{array}{cccc}
\left(\boldsymbol{C}_{v}+\boldsymbol{I}\right) a_{11}+\boldsymbol{I} d_{11} & \left(\boldsymbol{C}_{v}+\boldsymbol{I}\right) a_{12}+\boldsymbol{I} d_{12} & \cdots & \left(\boldsymbol{C}_{v}+\boldsymbol{I}\right) a_{1 N}+\boldsymbol{I} d_{1 N} \\
\left(\boldsymbol{C}_{v}+\boldsymbol{I}\right) a_{21}+\boldsymbol{I} d_{21} & \left(\boldsymbol{C}_{v}+\boldsymbol{I}\right) a_{22}+\boldsymbol{I} d_{22} & & \vdots \\
\vdots & & \ddots & \vdots \\
\left(\boldsymbol{C}_{v}+\boldsymbol{I}\right) a_{N 1}+\boldsymbol{I} d_{N 1} & \left(\boldsymbol{C}_{v}+\boldsymbol{I}\right) a_{N 2}+\boldsymbol{I} d_{N 2} & \cdots & \left(\boldsymbol{C}_{v}+\boldsymbol{I}\right) a_{N N}+\boldsymbol{I} d_{N N}
\end{array}\right]\right)+\beta^{1 / 2} \mu u_{0} \cos \omega \tau \times
\end{aligned}
$$




$$
\begin{aligned}
& +\left[\begin{array}{cccc}
\left(\boldsymbol{M}_{a}+\boldsymbol{I}\right) a_{11}-\boldsymbol{I} d_{11} & \left(\boldsymbol{M}_{a}+\boldsymbol{I}\right) a_{12}-\boldsymbol{I} d_{12} & \cdots & \left(\boldsymbol{M}_{a}+\boldsymbol{I}\right) a_{1 N}-\boldsymbol{I} d_{1 N} \\
\left(\boldsymbol{M}_{a}+\boldsymbol{I}\right) a_{21}-\boldsymbol{I} d_{21} & \left(\boldsymbol{M}_{a}+\boldsymbol{I}\right) a_{22}-\boldsymbol{I} d_{22} & & \\
\vdots & & \ddots & \\
\left(\boldsymbol{M}_{a}+\boldsymbol{I}\right) a_{N 1}-\boldsymbol{I} d_{N 1} & \left(\boldsymbol{M}_{a}+\boldsymbol{I}\right) a_{N 2}-\boldsymbol{I} d_{N 2} & \cdots & \left(\boldsymbol{M}_{a}+\boldsymbol{I}\right) a_{N N}-\boldsymbol{I} d_{N N}
\end{array}\right]+ \\
& +\beta^{1 / 2} \mu u_{0}\left(1-\frac{1}{2} \delta\right) \cos \omega \tau\left[\begin{array}{cccc}
\boldsymbol{I} b_{11} & \boldsymbol{I}_{1} b_{12} & \cdots & \boldsymbol{I} b_{1 N} \\
\boldsymbol{I} b_{21} & \boldsymbol{I} b_{22} & & \\
\vdots & & \ddots & \vdots \\
\boldsymbol{I} b_{N 1} & \boldsymbol{I} b_{N 2} & \cdots & \boldsymbol{I} b_{N N}
\end{array}\right],
\end{aligned}
$$

where the defining constant parameters in $\tilde{\boldsymbol{K}}_{1}(\tau)$ is $p=1 / 2(1-\delta) c_{b}+(1-1 / 2 \delta)\left[1 / 2 \varepsilon c_{f}(1+h)\right]$.

For convenience, introducing the state variable, $\boldsymbol{z}=[\boldsymbol{q} \dot{\boldsymbol{q}}]^{\boldsymbol{T}}$, the Eq. (36) is converted into the first order form as follows:

$$
\dot{z}=A z+B(\tau) z+Q
$$

where both dimensions of $\boldsymbol{A}$ and $\boldsymbol{B}$ are $4 K N \times 4 K N$. Their expressions of $\boldsymbol{A}, \boldsymbol{B}$ and $\boldsymbol{Q}$ are as:

$$
\begin{aligned}
& \boldsymbol{A}=\left[\begin{array}{cc}
0 & \boldsymbol{I} \\
-\tilde{\boldsymbol{M}}^{-1} \tilde{\boldsymbol{K}} & -\tilde{\boldsymbol{M}}^{-1} \tilde{\boldsymbol{C}}
\end{array}\right], \\
& \boldsymbol{B}(\tau)=\left[\begin{array}{cc}
0 & 0 \\
-\tilde{\boldsymbol{M}}^{-1} \tilde{\boldsymbol{K}}_{1} & -\tilde{\boldsymbol{M}}^{-1} \tilde{\boldsymbol{C}}_{1}
\end{array}\right], \\
& \boldsymbol{Q}=\left[\begin{array}{c}
0 \\
-\tilde{\boldsymbol{M}}^{-1} \tilde{\boldsymbol{Q}}
\end{array}\right] .
\end{aligned}
$$

\section{Results and discussion}

This section is mainly about the numerical calculation of the established model. The dynamic characteristics of a CB coupling system are studied by following example analysis.

\subsection{Model \& numerical method validation}

In order to verify the correctness of the model and analyze the stability, when the pulsating parameters is $\mu=0$ and the external generalized force is $\boldsymbol{Q}=\mathbf{0}$, the Eq.(38) can be transformed into ordinary differential equation $\dot{\boldsymbol{z}}=\boldsymbol{A} \boldsymbol{z}$. And its analytical solution can be expressed as:

$$
z=X e^{\mathrm{i} \omega \tau}
$$

Let $\lambda=\mathrm{i} \omega$, and substitute Eq.(40) into $\dot{\boldsymbol{z}}=\boldsymbol{A} \boldsymbol{z}$, the eigenvalue equation is obtained as:

$$
(\lambda \boldsymbol{I}-\boldsymbol{A}) \boldsymbol{X}=\boldsymbol{0} .
$$

Through solving the Eq. (41), the $4 K N$ eigenvalues and $4 K N$ eigenvectors can be obtained. If the complex number appears, it must conjugate in pairs. Any characteristic frequency with positive real part is denoted as $\omega_{i}=\bar{\omega}_{i}+\mathrm{i} \rho_{i}$ corresponding to the eigenvector $\boldsymbol{X}_{i}, \tilde{\boldsymbol{X}}_{i}$ consisting of $2 K N$ elements in the upper half part of the vector is expressed as $\tilde{\boldsymbol{X}}_{i l}$, which is corresponding to $\boldsymbol{q}_{l}$. Let $\tilde{\boldsymbol{X}}_{i l}=\boldsymbol{R}_{i l}+\mathrm{i} \boldsymbol{I}_{i l}$, combining Eq. (40) and Eq. (32), the displacement can be expressed as:

$$
\boldsymbol{\eta}(\xi, \tau)=\sum_{l=1}^{N} \phi_{l}(\xi) \sum_{i=1}^{2 K N} e^{-\rho_{i} \tau}\left[2 c_{i}\left(\boldsymbol{R}_{i l} \cos \bar{\omega}_{i} \tau-\boldsymbol{I}_{i l} \sin \bar{\omega}_{i} \tau\right)-2 d_{i}\left(\boldsymbol{R}_{i l} \sin \bar{\omega}_{i} \tau+\boldsymbol{I}_{i l} \cos \bar{\omega}_{i} \tau\right)\right]
$$

where the constants $c_{i}, d_{i}$ can be determined by the initial conditions.

Firstly, Argand diagram and natural frequency varying with gap are calculated and compared with Suss S. results to verify the model. The selected system parameters are the same as those selected by Suss S. [16]. Under steady flow, three cylinders' system with simply supports at both ends are analyzed. The values of the dimensionless parameters are as follows: $\beta=0.48, \varepsilon c_{f}=0.25, \delta=1, \alpha=\gamma=\Gamma=\Pi=0$, $G_{c}=G_{w}=0.25$.

As shown in Fig. 4, in order to be clearly visible, the values with zero real part are placed on the left side of the coordinate system. At lower velocity, all complex frequencies are only taken from the upper half plane of the complex plane. As can be seen from Fig.4, the critical velocity of first-order divergence buckling instability is $u_{\mathrm{c}} \approx$ 1.495 , and that of coupled mode flutter instability is 2.992 . The critical buckling instability velocity of a single cylinder in a sufficiently large channel is 3.145 , and the critical velocity coupling mode flutter is 6.41 . The comparison of the two cases shows that the critical velocity of instability is very low under such a small gap ratio in engineering. At the same flow velocity, the lowest modes of the first-order and second-order mode groups calculated in this paper are basically similar to the results calculated by Suss S. et al. [17]. As shown in Fig. 5, the four-cylinder bundle system has the identical parameter values of the three cylinders bundle system, except the parameter $\beta=0.1$. The effect of the gap ratio of the stationary fluid on the lowest modal frequency is studied. It is found that the effect of fluid coupling on natural frequencies decreases with the increase of the gap ratio. When the gap ratio is greater than $G_{c}>5$, the dimensionless natural frequencies tend to be constant 9.86, approximately equal to the natural frequency of a single cylinder in fluid, i.e. $\pi^{2}$. And the results are similar those of Suss S. Therefore, the above example verifies the correctness of the model and the numerical computational code. 


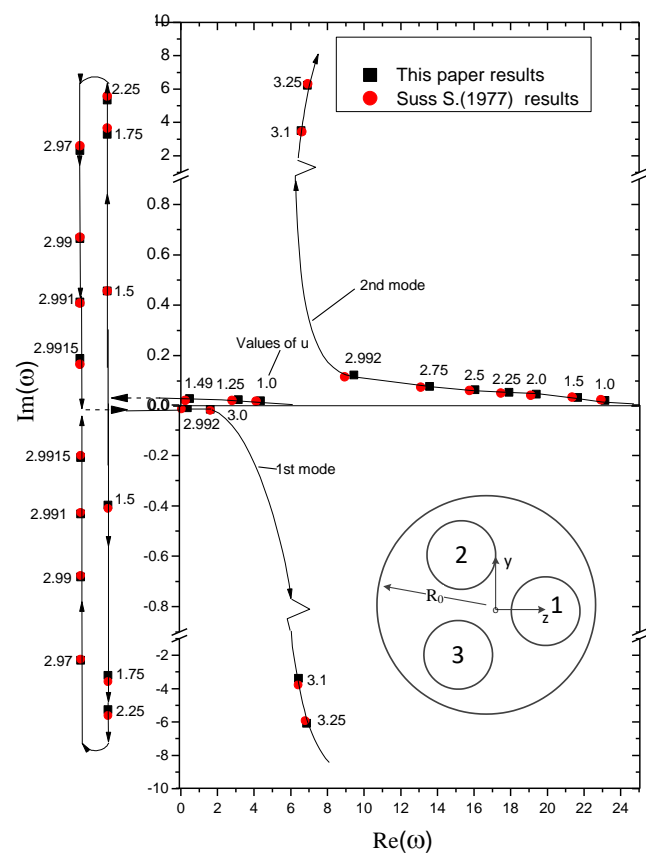

Fig. 4 Argand diagram of three cylinders' system

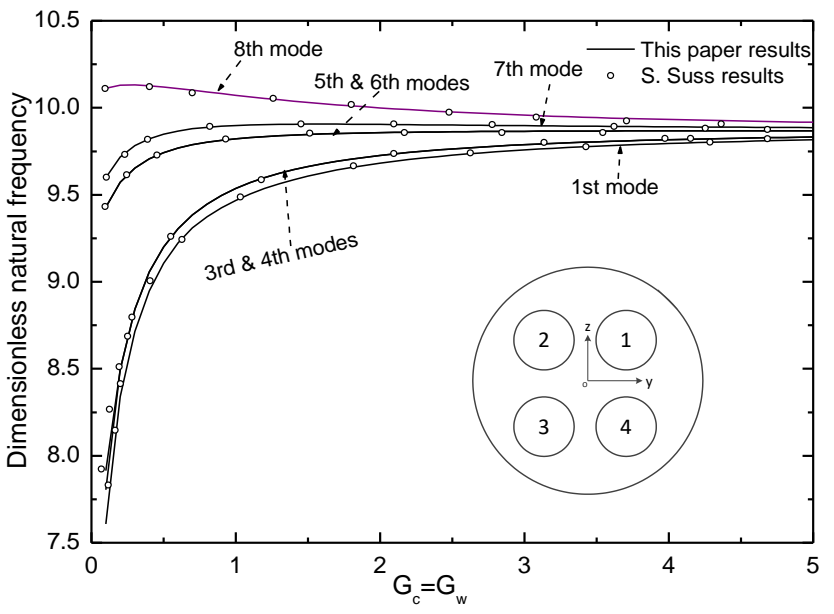

Fig. 5 Natural frequency of four cylinders' system with different gap ratio

\subsection{Analysis of engineering example}

Taking the design parameters of MYRRHA [23, 24] lead-based reactor as an example, the coolant is liquid metal lead-bismuth eutectic(LBE), the fuel is stored in stainless steel cladding tubes, and the rod bundles are positioned radially by wire wrapping the cladding. Simple support constraints are adopted at both ends of the axial direction. The fuel rod will elongate axially due to thermal expansion and other factors. If the downstream end is fixed, compression load will be generated and it is easier to lose stability. Therefore, the downstream end is designed to free sliding axially, i.e. $\delta=0$. Using liquid lead and bismuth as coolant, the fuel rod design parameters are as follows: the density of $316 \mathrm{~L}$ stainless steel cladding is $\rho_{\mathrm{c}}=7969.7 \mathrm{Kg} / \mathrm{m}^{\wedge} 3$, Yong modulus is $E=193.8 \mathrm{GPa}$, Poisson's ratio is $v=0.297$, the density of $\mathrm{UO}_{2}$ fuel is approximately $\rho_{\mathrm{UO} 2}=10000 \mathrm{Kg} / \mathrm{m}^{\wedge} 3$. fuel rod diameter is $D=6.55 \mathrm{~mm}$, the length is $L=1400 \mathrm{~mm}$, the cladding thickness is $0.51 \mathrm{~mm}$, Pitch is $P=8.35 \mathrm{~mm}$ and $P / D=1.27$. Other system parameters are shown in Table 1 .
For the small amplitude vibration of a three cylinders bundle system above, the Galerkin discrete order $N=3$ satisfies computational accuracy. Considering initial condition $z_{0}=[0 ; 0 ; 0 ; 0 ; 0 ; 0 ; 0 ; 0 ; 0 ; 0 ; 0 ; 0 ; 0 ; 0 ; 0 ; 0 ; 0 ; 0 ; 0 ; 0.01 ; 0.01 ;-$ $0.001 ;-0.001 ; 0 ; 0 ; 0 ; 0 ; 0 ; 0 ; 0 ; 0 ; 0 ; 0 ; 0 ; 0 ; 0]$ and substituting it into Eq. (42), the constants $c_{\mathrm{i}}, d_{\mathrm{i}}$ can be determined. The following is the comparison between the analytical solution and the numerical solution of the ordinary differential equation. The variable step size ode45 function based on MATLAB software is used to verify the accuracy and reliability of the Runge-Kutta method for solving the cylinders' bundle coupling dynamic equation. When the pulsating parameters is not zero, the analytical solution of Eq. (12) is difficult to obtain due to the existence of transient term $\boldsymbol{B}(\tau)$, but the Runge-Kutta method provides a numerical solution for the next section to calculate and solve the dynamic equation of rod bundle under pulsating flow.

As shown in Fig. 6, the horizontal free vibration of cylinder 1 was calculated using the analytical solution and the numerical method respectively. It can be seen that the two solutions are basically consistent, which verifies the accuracy of the calculation results of Runge-Kutta method.

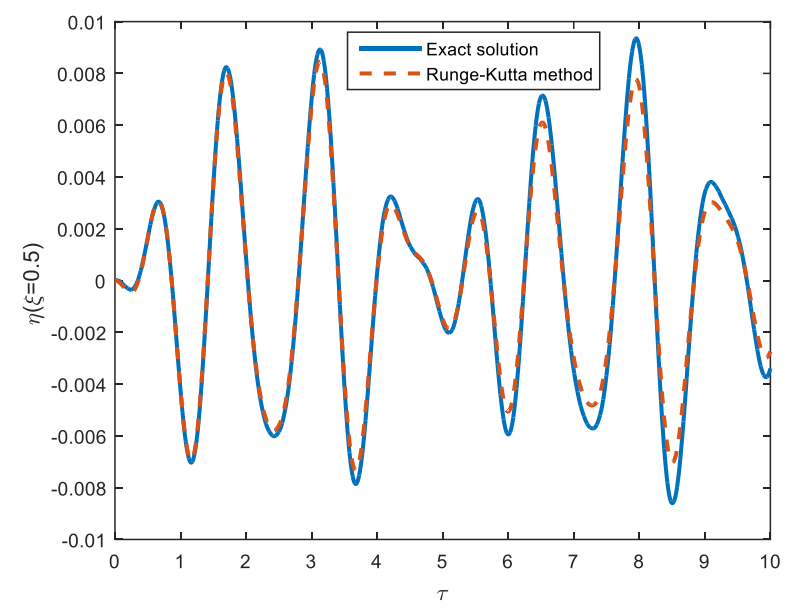

Fig. 6 The comparison between exact solution and numerical solution with $u=1.1$

In order to evaluate the uncertainty of dynamic changes of reactor operation system, three key parameters need to be analyzed, i.e. equivalent mass $m_{e}$, slenderness ratio $\varepsilon$, and dimensional velocity $U$. At the beginning of operation, the equivalent mass only contributes to the cladding, $m_{e}=m_{c}$, and at the middle and later stages, the equivalent mass includes the contribution of pellet, i.e. $m_{e}=m_{c}+m_{p}$ [26], in which $m_{c}$ is the cladding mass of unit length and $m_{\mathrm{p}}$ is the pellet mass of unit length. The slenderness ratio will also be discussed in terms of the length of a pitch or the total length of the pitch. Therefore, these changes will produce four combinations of parameters: $1 . m_{c}, \varepsilon=L / D, 2 . m_{c}, \varepsilon=H / D, 3$. $m_{c}+m_{p}, \varepsilon=L / D$, and 4. $m_{c}+m_{p}, \varepsilon=H / D$.

The natural frequency range in De Pauw's LBE experiment is 5.5-7.4 Hz [23]. As shown in Fig. 7, it is in the range of the natural frequencies based on the above two slenderness ratios. Because there is a gap between the cylinders in the position where the wire is crossing, the experiment value is closer to the frequency calculated in the case 3 , although the turbulence caused by wire spacer has an ef- 
fect on the static deformation, the dynamic response is similar to the bare fuel rod [27]. And thus the calculated results are reasonable.

\subsection{Numerical results of CB coupling system for forced vi- bration}

The forced vibration characteristics caused by the pulsating velocity parameters are calculated below. The system parameters are selected as the same as the seven $\mathrm{CB}$ system, and the slenderness ratio and the equivalent mass corresponding to the above case 3 are adopted. According to the MYRRHA project, the average lead-bismuth flow rate is $2 \mathrm{~m} / \mathrm{s}$ [24]. The external excitation along y direction, which is $g_{1} \sin \left(\omega^{\prime} \tau\right)$, is imposed on cylinder 1 and uniform distribution along axial direction, where the excitation frequency is $\omega^{\prime}=10$. The dimensionless pulsation velocity at the inlet is $u=u_{0}\left(1+\sin \omega_{0} \tau\right)$, where $u_{0}$ corresponds to $U_{0}=2 \mathrm{~m} / \mathrm{s}$.

For the sake of comparison, the magnification factor is defined as the ratio of the displacement at the middle span of any cylinder to the displacement at the middle span of cylinder 1 caused by the static load of excitation amplitude $\left(g_{1}\right)$.

In order to analyze the influence of the pulsating parameters on the system, firstly, the eigenfrequencies is given as Table 2 .

Table 1

System parameters $[24,25]$ used in calculations

\begin{tabular}{|l|l|l|l|}
\hline $\begin{array}{l}\text { Parameter } \\
\text { name }\end{array}$ & Value & $\begin{array}{l}\text { Parameter } \\
\text { name }\end{array}$ & Value \\
\hline $\begin{array}{l}\text { Flexural ri- } \\
\text { gidity, } E I\end{array}$ & $8.6135{\mathrm{~Pa} . \mathrm{m}^{\wedge} 4}^{4}$ & $\begin{array}{l}\text { LBE den- } \\
\text { sity }\end{array}$ & $10364 \mathrm{Kg} / \mathrm{m}^{\wedge} 3$ \\
\hline $\begin{array}{l}\text { Axial tension, } \\
\bar{T}\end{array}$ & $0.0 \mathrm{~N}$ & Pitch, $H$ & $265 \mathrm{~mm}$ \\
\hline$C_{f}$ & 0.02 & $C_{b}$ & 0.0 \\
\hline$C_{D}$ & 0.0 & $\delta$ & 0 \\
\hline
\end{tabular}

Table 2

Eigenfrequencies of the first mode group

\begin{tabular}{|c|c|c|c|c|c|c|c|c|c|}
\hline$n$ & $\omega_{1}, \omega_{2}$ & $\omega_{3}$ & $\omega_{4}$ & $\omega_{5}, \omega_{6}$ & $\omega_{7}, \omega_{8}$ & $\omega_{9}, \omega_{10}$ & $\omega_{11}$ & $\omega_{12}, \omega_{13}$ & $\omega_{14}$ \\
\hline Freq. & 6.985 & 6.991 & 7.489 & 7.592 & 7.666 & 9.844 & 10.423 & 11.721 & 12.273 \\
\hline
\end{tabular}

\subsection{Discussion}

As can be seen from Fig. 7, the critical flow velocity of instability calculated under the worst working condition (i.e. case 3 ) is about $13.4 \mathrm{~m} / \mathrm{s}$. Therefore, the flow rate of coolant designed by MYRRHA project will not cause instability under this working condition.

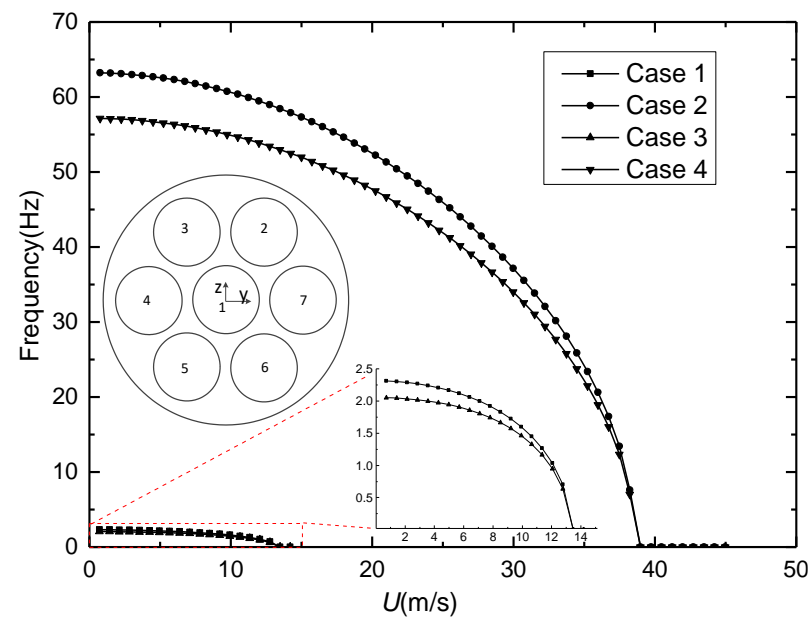

Fig. 7 Natural frequency variation with flow velocity

Fig. 8 shows the free vibration trajectories of each cylinder under different initial conditions of seven-cylinder

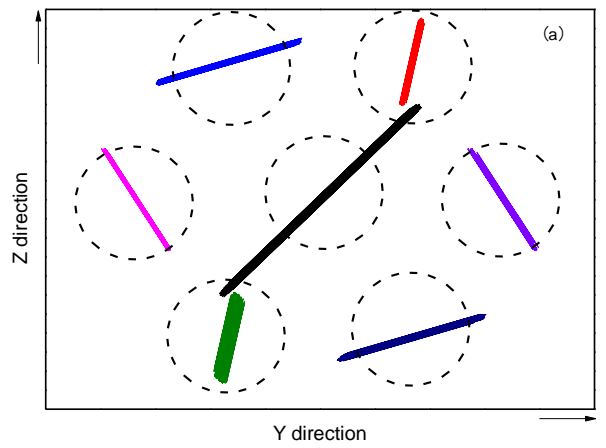

bundle. It can be seen that each cylinder is basically a plane motion. The motion trajectory directions in Fig. 8, a and Fig. 8, b are related to the two different initial conditions and the lowest cross-section vibration modal shapes. In this paper, the trajectories of stable motion under many different initial conditions are calculated, and it is found that they all mainly appear in one of the two motion modes such as Fig. 8, a or Fig. 8, b. It is also found that the motion mode of Fig. 8, a corresponds to the mode shape of multiple frequencies, while the motion mode shown in Fig. 8, b corresponds to the modal shape of single frequency in the first mode group. Because of the symmetry of the system, the following analysis only takes the motion of cylinder 2 in the $\mathrm{y}$ direction and $\mathrm{z}$ direction as an example.

As shown in Fig. 9, a, the peak amplitude corresponding to the frequency is about $\omega \approx 10$, the value is equal to excitation frequency just right, which satisfies the characteristic of forced vibration. The pulsating frequency parameter $\omega_{0}$ within the range $(1,2)$ has an influence on the amplitude and presents multi-peak characteristics, which are mainly caused by the coupling between the pulsating frequency, the characteristic frequency of the cylinder bundle system and the excitation frequency of cylinder 1. From Fig. 9, b, we can see that the amplification factor is the largest at $\omega_{0} \approx 1.5$, indicating that the coupling effect is the strongest under that frequency.

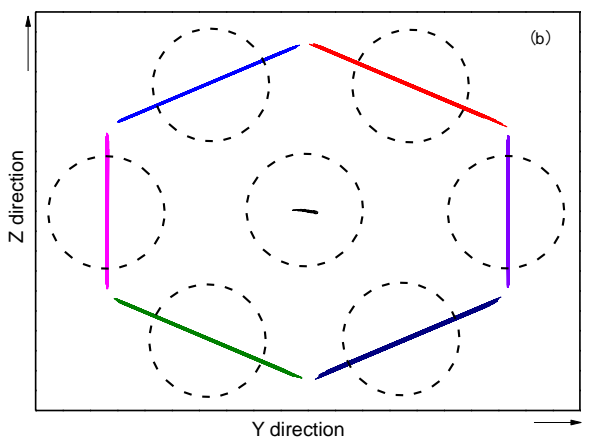

Fig. 8 Free vibration locus at $\xi=0.5$ plane under steady flow $U_{0}=2 \mathrm{~m} / \mathrm{s}$ in two different initial conditions 
As shown in Fig. 10, the amplitude amplification factor increases linearly with the pulsating parameter $\mu$. As shown in Fig. 11, the time history of displacement at three different pulsating frequencies shows that the time history displacement of $\omega_{0}=10$ is almost coincident with the time history displacement of steady flow, i.e. $\omega_{0}=0$, indicating that the pulsating frequency has almost no influence on the forced vibration system with the increase of the pulsating frequency.

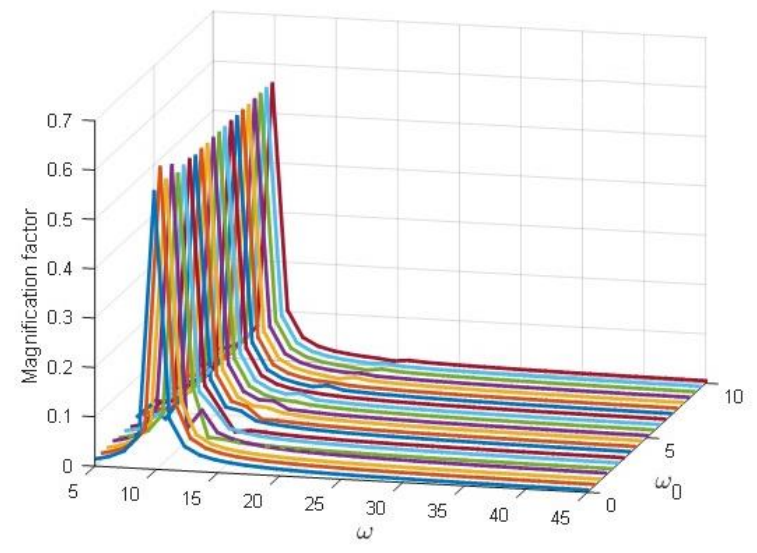

a
Fig. 12 shows the cross section motion trajectory of axis midpoint of the CB system when $U_{0}=2 \mathrm{~m} / \mathrm{s}$ and $\mu=0.2$. Compared with Fig. 8, we know that forced vibration has a great influence on the motion trajectory direction, but it is still a plane motion. Because of the symmetry of the structure, the trajectory of the $\mathrm{CB}$ caused by forced vibration has symmetry.

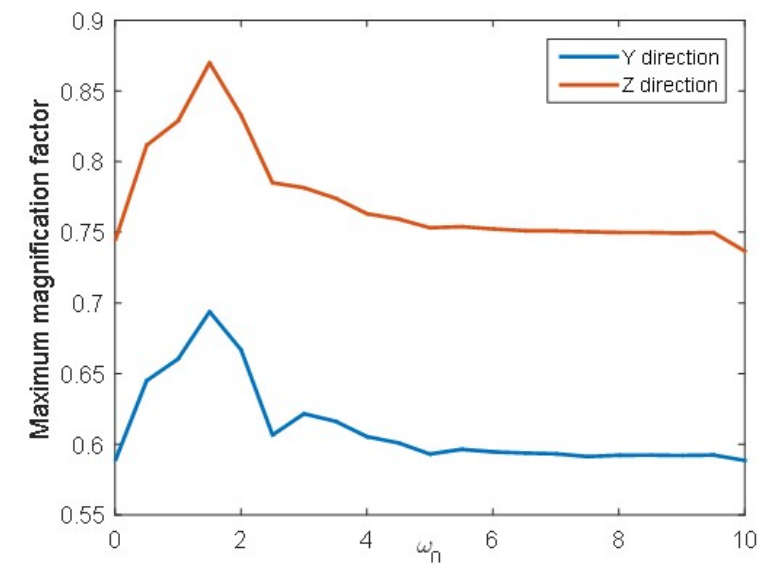

$\mathrm{b}$

Fig. 9 a) Amplitude frequency response of cylinder 2 in y direction at different pulsating frequencies $\omega_{0}$ with $U_{0}=2.0 \mathrm{~m} / \mathrm{s}$, $\mu=0.2$; b) Maximum magnification factor in $y$ direction and in $\mathrm{z}$ direction with the pulsating frequencies varying

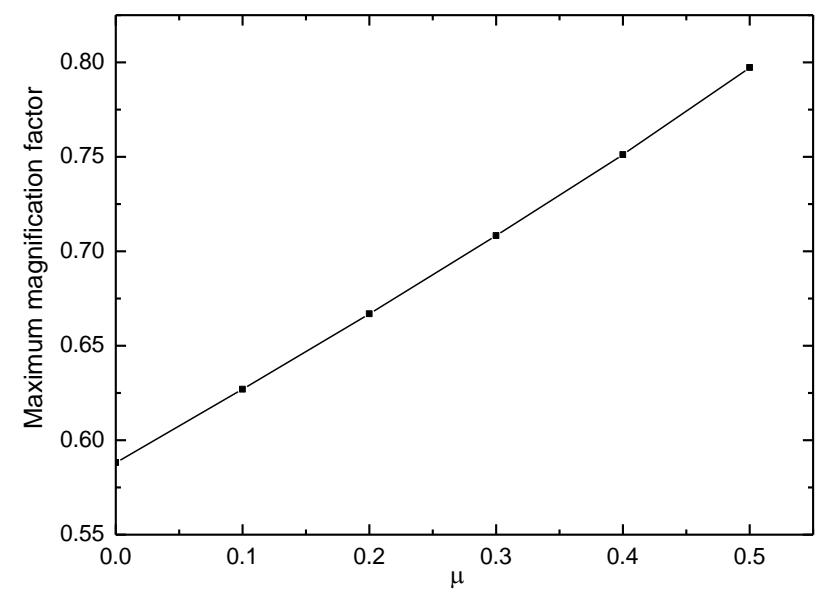

Fig. 10 Effect of pulsating parameter $\mu$ on the amplitude of forced vibration with $U_{0}=2 \mathrm{~m} / \mathrm{s}, \omega_{0}=2$

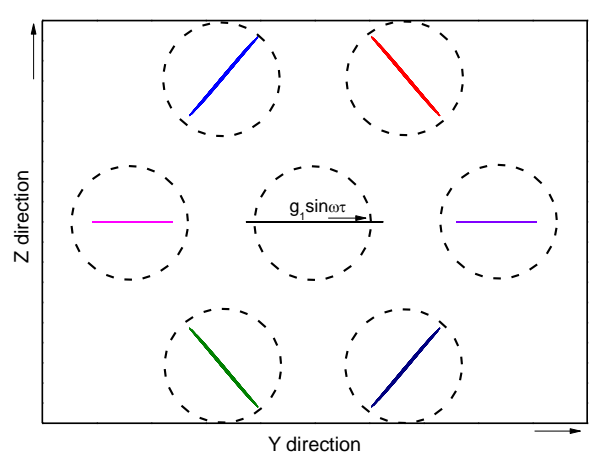

Fig. 12 Motion locus of seven cylinders bundle system subjected to pulsating flow forced vibration with $U_{0}=2 \mathrm{~m} / \mathrm{s}, \mu=0.2, \omega_{0}=2$

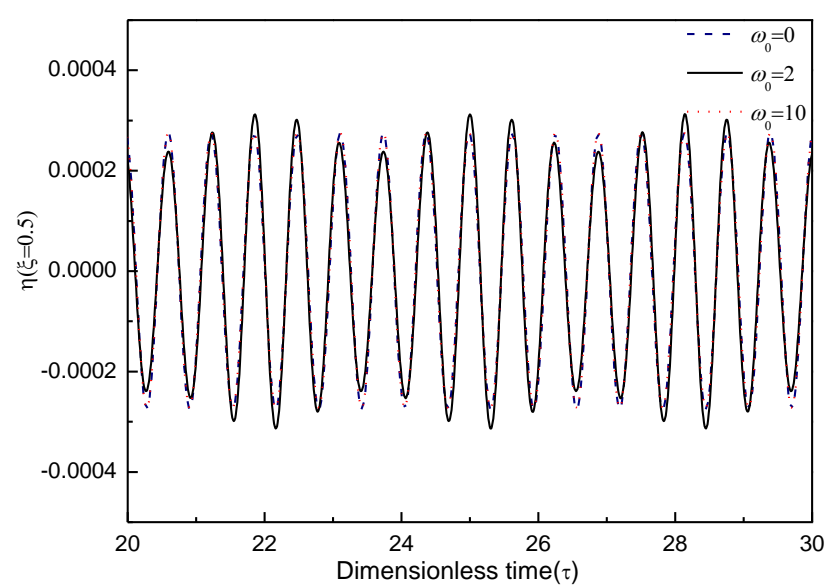

Fig. 11 Dimensionless dynamical deflections of cylinder 2 in y direction change over time at three different pulsating frequencies with $U_{0}=2 \mathrm{~m} / \mathrm{s}, \mu=0.2$

\section{Conclusion}

Based on the modeling ideas of Chen and Païdoussis, the dynamic model of cylinders bundle forced vibration system under axial pulsating flow is established in this paper. The eigenvalues of the linear system and the pulsating parameters effect on the vibration amplitude are calculated and analyzed, the following conclusions are obtained:

1) It can be seen from Argand diagram that the state of the system changes from stable to unstable with the increase of flow velocity. And the dimensionless natural frequency of cylinders bundle system tends to the natural frequency $\pi^{2}$ of a single cylinder system with the increase of gap ratio.

2) The natural frequency decreases with the increase of the flow velocity, and the slope of the change of frequency also increases, indicating that the higher the flow velocity is 
before the instability, the more significant the influence on the natural frequency of the system is.

3) In MYRRHA project, the critical flow velocity of instability is $13.4 \mathrm{~m} / \mathrm{s}$. Therefore, the designed flow velocity limit of $2 \mathrm{~m} / \mathrm{s}$ is far lower than the critical flow velocity of instability, and thus the fuel assembly will not experience buckling instability.

4) It finds that the pulsating parameters, $\omega_{0}$, has the strongest cylinders bundle coupling effect within the range $(1.0,2.0)$, and the vibration amplitude increases linearly with parameter $\mu$.

\section{Acknowledgments}

This work is supported by the Strategic Priority Research Program of Chinese Academy of Sciences, Grant No. XDA21010202.

\section{Reference}

1. Pettigrew, M. J.; Taylor, C. E.; Fisher N. J.; Yetisir M.; Smith, B. A.W. 1998. Flow-induced vibration: recent findings and open questions, Nuclear Engineering and Design 185(2-3): 249-276. https://doi.org/10.1016/S0029-5493(98)00238-6.

2. Ridder De, J.; Degroote, J.; Van Tichelen, K.; Vierendeels, J. 2017. Predicting modal characteristics of a cluster of cylinders in axial flow: From potential flow solutions to coupled CFD-CSM calculations, Journal of Fluids and Structures 4: 90-110.

https://doi.org/10.1016/j.jfluidstructs.2017.07.006.

3. Xie, T.; Liu, J. H.; Wang, H. K.; Hai, H. T.; Du, D.; Yan, B. 2019. Added mass coefficient of elastic rods in cylindrical fluid, Nuclear Engineering and Design 342:249-256.

https://doi.org/10.1016/j.nucengdes.2018.12.010.

4. Zeman, V.; Dyk, Š.; Hlaváč, Z. 2016. Mathematical modeling of nonlinear vibration and fretting wear of the nuclear fuel rods, Archive of Applied Mechanics 86(4): 657-668. https://doi.org/10.1007/s00419-015-1053-0.

5. Tang, L. C.; Ding, S. R.; Qian, H.; Xie, Y. C.; Huo, Y. Z. 2014. Fretting fatigue tests and crack initiation analysis on zircaloy tube specimens, International Journal of Fatigue 63:154-161.

https://doi.org/10.1016/j.ijfatigue.2014.01.020.

6. Chen, S. S. 1975. Vibration of nuclear fuel rods, Nuclear Engineering and Design 35:399-422. https://doi.org/10.1016/0029-5493(75)90071-0.

7. Païdoussis, M. P. 2004. Fluid-Structure interactions: slender structures and axial flow, vol. 2, Academic Press Limited, London.

8. Païdoussis, M. P.; Suss, S. 1977. Stability of a cluster of flexible cylinder in bounded axial flow, Journal of Applied Mechanics 44:401-408. https://doi.org/10.1115/1.3424091.

9. Liu, Z. G.; Liu, Y.; Lu, J. 2013. Numerical simulation of the fluid-structure interaction for two simple fuel assemblies, Nuclear Engineering and Design 258: 1-12. https://doi.org/10.1016/j.nucengdes.2013.01.029.

10. Jin, J. D.; Song, Z. Y. 2005. Parametric resonances of supported pipes conveying pulsating fluid, Journal of Fluids and Structures 20(6):763-783.

https://doi.org/10.1016/j.jfluidstructs.2005.04.007.
11. Wang, L. 2005. Stability, bifurcations, and chaos in pipes conveying fluid, Ph.D. thesis, Huazhong University of Science \& Technology, Wuhan, China.

12. Païdoussis, M. P. 1975. Stability of flexible slender cylinders in pulsatile axial flow, Journal of Sound and Vibration 42(1): 1-11.

13. https://doi.org/10.1016/0022-460X(75)90298-9.

14. Païdoussis, M. P.; Suss, S. 1977. Stability of a cluster of flexible cylinders in bounded axial flow, Journal of Applied Mechanics 44(3): 401-408. https://doi.org/10.1115/1.3424334.

15. Païdoussis, M. P. 1973. Dynamics of cylindrical structures subjected to axial flow, Journal of Sound and Vibration 29: 365-385. https://doi.org/10.1016/S0022-460X(73)80291-3.

16. Chen, S. S.; Wambsganss, M. W. 1972. Parallel-flowinduced vibration of fuel rods, Nuclear Engineering and Design 8(2): 253-278. https://doi.org/10.1016/0029-5493(72)90144-6.

17. Lighthill, M. J. 1960. Note on the swimming of slender fish, Journal of fluid Mechanics 9(02): 305-317. https://doi.org/10.1017/S0022112060001110.

18. Currie, I. G. 2012. Fundamental mechanics of fluids, 3rd Edition, McGraw Hill Inc.

19. Suss, S. 1977. Dynamics of clusters of flexible cylinders in bounded axial fluid flow. M. Eng. Thesis, McGill University, Montreal, Canada.

20. Taylor, G. 1952. Analysis of the swimming of long and narrow animals, Proceedings of the Royal Society of London Series A-Mathematical Physical and Engineering Sciences 214 (1117): 158-183. http://www.jstor.org/stable/99081.

21. Modarres Sadeghi, Y.; Païdoussis, M. P.; Semler C. 2005. A nonlinear model for an extensible slender flexible cylinder subjected to axial flow, Journal of Fluids and Structures 21(5): 609-627. https://doi.org/10.1016/j.jfluidstructs.2005.02.012.

22. Païdoussis, M. P.; Issid N. T. 1974. Dynamic stability of pipes conveying fluid, Journal of Sound and Vibration 33(3): 267-294. https://doi.org/10.1016/S0022-460X(74)80002-7.

23. Uitslag-Doolaard, H. J., Roelofs, F., Pacio, J. C. 2019. Experiment design to assess the inter-wrapper heat transfer in LMFR, Nuclear Engineering and Design 341:297-305. https://doi.org/10.1016/j.nucengdes.2018.11.019.

24. De Pauw, B. 2015. Experimental analysis of flow-induced vibration in Lead-Bismuth Cooled Nuclear Fuel Assemblies, Ph.D. thesis, Vrije Universiteit Brussel, Brussels, Belgium.

25. Santis De, D., 2017. Numerical simulations of rod assembly vibration induced by turbulent axial flow, $\mathrm{Nu}-$ clear Engineering and Design 320:44-56. https://doi.org/10.1016/j.nucengdes.2018.04.027.

26. De Pauw, B.; Weijtjens, W.; Vanlanduit, S., et al., 2015. Operational modal analysis of flow-induced vibration of nuclear fuel rods in a turbulent axial flow, $\mathrm{Nu}-$ clear Engineering and Design 284:19-26. https://doi.org/10.1016/j.nucengdes.2014.11.040.

27. Kim, H. K.; Kim, M. S. 2005, Vibration analysis of PWR fuel rod, Journal of Sound and Vibration 282(1-2): 553-572. https://doi.org/10.1016/j.jsv.2004.04.024. 
28. De Santis, D.; Shams, A. 2019, Analysis of flow induced vibrations and static deformations of fuel rods considering the effects of wire spacers and working fluids, Journal of Fluids and Structures 84: 440-465. https://doi.org/10.1016/j.jfluidstructs.2018.11.015

Y. Shu, J. Wu, Y. Yang, W. Liu, K. Tao

\section{DYNAMIC CHARACTERISTICS ANALYSIS OF CYLINDERS BUNDLE COUPLING SYSTEM UNDER AXIAL FLOW}

S u m m a r y

Nuclear reactor fuel assemblies are mainly composed of cylinders bundle(CB), calculating the dynamics characteristics of $\mathrm{CB}$ under axial flow can lay a foundation for predicting fretting wear and vibration fatigue. In the paper, the CB coupling dynamic model of forced vibration under pulsating flow is established. And the stability analysis and natural frequency calculation of the CB system under steady flow are compared with the existing results to verify the model. Finally, the Runge-Kutta method is applied to solve the forced vibration equation of the CB under pulsating flow. The influence of the pulsating parameters $\mu, \omega_{0}$ on the amplitude-frequency characteristics and the motion trajectory of the midspan cross section of the CB under forced vibration are analyzed and discussed. The results show that the pulsating parameters have an important influence on the vibration of the $\mathrm{CB}$ system.

Keywords: CB coupling dynamic model, stability analysis, pulsating flow, amplitude frequency response, movement trajectory.

Received January 19, 2020

Accepted June 02, 2021

This article is an Open Access article distributed under the terms and conditions of the Creative Commons Attribution 4.0 (CC BY 4.0) License (http://creativecommons.org/licenses/by/4.0/). 